\title{
Role of Williamsia and Segniliparus in human infections with the approach taxonomy, cultivation, and identification methods
}

\author{
Mehdi Fatahi-Bafghi* (i)
}

\begin{abstract}
The genera Williamsia and Segniliparus are of aerobic actinomycetes and at the time of writing, they have 12 and 2 species, respectively. These genera cause various infections in humans. In this review, we surveyed their taxonomy, isolation, identification, as well as their role to cause human infections.
\end{abstract}

Keywords: Actinomycete, Isolation, Taxonomy, Segniliparus, Williamsia

\section{Introduction}

Aerobic actinomycetes are the group of Gram-positive bacilli belonging to the phylum Actinobacteria. Some species that cause human infections in this group are situated in one of the four suborders, including Corynebacterineae, Micrococcineae, Streptomycineae and Streptosporangineae [1]. Kämpfer et al. and Butler et al. suggested that the genera of Williamsia [2] and Segniliparus that belong to the actinomycete family, [3] respectively, can cause human infections. They non-spore, non-motile aerobic organisms with short rods without branching that contain mycolic acid components in the cell wall structure [4]. DNA G+C content in the genera Williamsia and Segniliparus are 64-65\% and 68-72\% [4] respectively, and they are into the order Corynebacteriales (Tindall [5] proposed the name of Corynebacteriales to be replaced by Mycobacteriales) and suborder Corynebacterineae. The various genera include Corynebacterium, Dietzia, Hoyosella, Gordonia, Lawsonella, Millisia, Mycobacterium, Mycobacteroides, Mycolicibacillus, Mycolicibacter, Mycolicibacterium, Nocardia, Rhodococcus, Skermania, Smaragdicoccus,

\footnotetext{
*Correspondence: mehdifatahi@ssu.ac.ir
}

Shahid Sadoughi University of Medical Sciences and Health Services, Yazd, Iran
Tomitella and, Tsukamurella are located in this suborder (https://www.ncbi.nlm.nih.gov/Taxonomy/Browser/ wwwta $x$.c gi ? mode $=$ Undef \& id $=85007$ $\& \mathrm{lvl}=3 \&$ lin $=\mathrm{f} \& \mathrm{keep}=1 \&$ srchmode $=1 \&$ unlock). To date, various infections cause by actinomycetes are on the rise. The most common genera that cause infections in this suborder include Corynebacterium (such as Corynebacterium accolens [6], Corynebacterium afermentans [6], Corynebacterium amycolatum [6], Corynebacterium appendicis [7], Corynebacterium argentoratense [7], Corynebacterium aurimucosum [6], Corynebacterium coyleae [7], Corynebacterium diphtheriae bv. mitis [6], Corynebacterium durum [7], Corynebacterium freneyi [7], Corynebacterium glucuronolyticum [6], Corynebacterium hansenii [7], Corynebacterium imitans [6], Corynebacterium jeikeium [6], Corynebacterium kroppenstedtii [8], Corynebacterium lipophiloflavum [7], Corynebacterium macginleyi [6], Corynebacterium massiliense [7], Corynebacterium minutissimum [6], Corynebacterium mucifaciens [6], Corynebacterium mycetoides [7], Corynebacterium pseudodiphtheriticum [6], Corynebacterium pilbarense [7], Corynebacterium propinquum [6], Corynebacterium pyruviciproducens [7], Corynebacterium riegelii [7], Corynebacterium resistans [7], Corynebacterium simulans [6], Corynebacterium singular [7], Corynebacterium sputi [7], Corynebacterium 
stationis [7], Corynebacterium striatum [6], Corynebacterium sundsvallense [7], Corynebacterium thomsenii [7], Corynebacterium timonense [7], Corynebacterium tuberculostearicum [6], Corynebacterium tuscaniense [7], Corynebacterium ureicelerivorans [6]), Gordonia (such as Gordonia aichiensis [9], Gordonia amicalis [9], Gordonia araii [9], Gordonia bronchialis [9], Gordonia effuse [9], Gordonia otitidis [9], Gordonia polyisoprenivorans [9], Gordonia sputi [9], Gordonia terrae [9], Gordonia westfalica [10]), Mycobacterium (such as Mycobacterium abscesus [11], Mycobacterium ahvazicum [12], Mycobacterium alsense [13], Mycobacterium alsiense [14], Mycobacterium arupense [15], Mycobacterium avium [11], Mycobacterium bacteremicum [14], Mycobacterium barrassiae [14], Mycobacterium bouchedurhonense [14], Mycobacterium canettii [16], Mycobacterium celeriflavum [14], Mycobacterium chelonae [11], Mycobacterium chimaera [17], Mycobacterium conceptionense [18], Mycobacterium engbaekii [14], Mycobacterium europaeum [14], Mycobacterium flavescens [19], Mycobacterium fortuitum [11], Mycobacterium fragae [14], Mycobacterium franklinii [14], Mycobacterium fukienense [14], Mycobacterium gadium [19], Mycobacterium gordonae [11], Mycobacterium heckeshornense [20], Mycobacterium heraklionense [14], Mycobacterium immunogenum [21], Mycobacterium insubricum [14], Mycobacterium intracellulare [11], Mycobacterium iranicum [14], Mycobacterium malmoense [22], Mycobacterium mucogenicum [11], Mycobacterium kansasii [11], Mycobacterium koreense [14], Mycobacterium kumamotonense [14], Mycobacterium kyorinense [14], Mycobacterium lentiflavum [11], Mycobacterium lepromatosis [14], Mycobacterium llatzerense [14], Mycobacterium longobardum [14], Mycobacterium mageritense [23], Mycobacterium mantenii [14], Mycobacteriummarinum [24], Mycobacterium marseillense [14], Mycobacterium monacense [14], Mycobacterium novocastrense [19], Mycobacterium noviomagense [14], Mycobacterium orygis [14], Mycobacterium paraffinicum [14], Mycobacterium paragordonae [14], Mycobacterium parakoreense [14], Mycobacterium paraseoulense [14], Mycobacterium paraterrae [14], Mycobacterium peregrinum [19], Mycobacterium porcinum [19], Mycobacterium riyadhense [14], Mycobacterium scrofulaceum [11], Mycobacterium senuense [14], Mycobacterium seoulense [14], Mycobacterium setense [14], Mycobacterium sherrisii [14], Mycobacterium shigaense [14], Mycobacterium shinjukuense [14], Mycobacterium simiae [25], Mycobacterium simulans [14], Mycobacterium sinense [14], Mycobacterium thermoresistibile [18], Mycobacterium timonense [14], Mycobacterium tuberculosis [26], Mycobacterium ulcerans [27], Mycobacterium vulneris [14], Mycobacterium xenopi [11], Mycobacterium yongonense [14]), Tsukamurella (such as Tsukamurella asaccharolytica [28], Tsukamurella conjunctivitidis [28], Tsukamurella hongkongensis [29], Tsukamurella inchonensis [29], Tsukamurella paurometabola [29], Tsukamurella pseudospumae [29], Tsukamurella pulmonis [29], Tsukamurella serpentis [29], Tsukamurella sinensis [29], Tsukamurella soli [29], Tsukamurella spumae [29], Tsukamurella sputi [28], Tsukamurella strandjordae [29], Tsukamurella tyrosinosolvens [29]), Nocardia (such as Nocardia abscessus [30], Nocardia amamiensis [30], Nocardia amikacinitolerans [31], Nocardia araoensis [30], Nocardia arthritidis [30], Nocardia asiatica [30], Nocardia asteroides [30], Nocardia barduliensis [32], Nocardia beijingensis [30], Nocardia blacklockiae [33], Nocardia boironii [30], Nocardia brasiliensis [30], Nocardia caviae [30], Nocardia carnea [34], Nocardia cerradoensis [30], Nocardia colli [35], Nocardia concava [30], Nocardia crassostreae [30], Nocardia cyriacigeorgica [30], Nocardia exalbida [30], Nocardia farcinica [30], Nocardia gipuzkoensis [32], Nocardia harenae [30], Nocardia higoensis [30], Nocardia ignorata [36], Nocardia kruczakiae [30], Nocardia mexicana [30], Nocardia neocaledoniensis [30], Nocardia nova [30], Nocardia otitidiscaviarum [30], Nocardia paucivorans [30], Nocardia pseudobrasiliensis [30], Nocardia puris [30], Nocardia takedensis [30], Nocardia thailandica [30], Nocardia transvalensis [30], Nocardia veterana [30], Nocardia wallacei [37], Nocardia yamanashiensis [30]) and, Rhodococcus (such as Rhodococcus equi \{renamed to prescottella equi\} [38], Rhodococcus erythropolis [38], Rhodococcus ruber [38], Rhodococcus gordoniae [38], Rhodococcus facsians [38]). This study was performed because of the lack of attention and awareness of physicians to infections caused by these bacteria and to inform medical laboratory personnel about the methods of isolation and detection of these bacteria at the genus and species level. Our literature review focused on the human infections caused by Williamsia andSegniliparus considering taxonomy, cultivation, and identification methods through searching four databases, including Google Scholar, PubMed, Scopus, and Web of Science up to Oct 28, 2020, for all articles in English language, such as case reports, original articles, review article and books were 7, 17, 2, and 3 articles respectively.

\section{Cell wall structure in Williamsia}

The genera Williamsia and Segniliparus has a wall chemotype IV [4, 39]. In the cell wall, Williamsia contains meso-2,6-diaminoheptanedioate $\left(\mathrm{C}_{7} \mathrm{H}_{14} \mathrm{~N}_{2} \mathrm{O}_{4}\right)$, dihydrogenated menaquinone with nine isoprene units (Williamsia deligens has dihydrogenated menaquinone with eight isoprene units [40]), diphosphatidylglycerol, tuberculostearic acids, phosphatidylethanol, phosphatidylglycerol, $\mathrm{N}$-glycolyl muramic acid, phosphatidylinositol 
and, mycolic acids [4]. Muramic acid is glycosylated in the genera of Tsukamurella, Tomitella, Smaragdicoccus, Skermania, Rhodococcus, Nocardia, Mycobacterium, Millisia, and Gordonia, but it is acetylated in the Dietzia and Corynebacterium [4]. The fatty acids of Williamsia are hexadecenoic acid (C16:1-trans) oleic acid (C18:1), palmitic acid (C16:0), and tuberculostearic acid (10-methyl octadecanoate) [4]. In the Williamsia, some carbons in chain mycolic acids are C50-C56 [41].

\section{Cell wall structure in Segniliparus}

The cell wall of Segniliparus contains meso-diaminopimelic acid, mycolic acids and, tuberculostearic acid [3]. The fatty acids of Segniliparus are C10:0, C14:0, C16:0, and tuberculostearic acid [3]. In the Segniliparus, some carbons in chain mycolic acids are C60-C100 [42], but in the other genera such as Nocardia, Skermania, Gordonia, Tsukamurella, Mycobacterium, Millisia, Rhodoccocus, Dietzia, Hoyosella, and Corynebacterium they are C46-C60, C58-C64, C46-C66, C64-C78, C60-C90, C44-C52, C30-C54, C34-C38, C30-C35 and, C22-C36 [41] respectively.

\section{Isolation methods for Williamsia spp.}

Collection and transportation of clinical specimens to the medical laboratory are two important principles in the isolation of aerobic actinomycetes from the infections [1]. At the time of writing, the specific media have not been described for the isolation of Williamsia from human clinical samples. In literature, various media have been used for Williamsia isolation from various sources; however, those associated with good growth or appropriate for morphological examination are columbia agar supplemented with $5 \%$ sheep blood agar and brain heart infusion (BHI) agar [40, 43], M3 agar supplemented with cycloheximide and nystatin [44], glucose/yeast extract agar (GYEA) plates [44, 45], raffinose-histidine agar plate supplemented with cycloheximide and nystatin [45], tryptic soy agar (trypticase soy agar/tryptone soy agar) [2, 46-48], starch-casein agar supplemented with cycloheximide [47], nystatin and rifampicin and ISP media 2-7 [47], modified Bennett's agar [47], glucose-yeast extract malt extract agar [47], nutrient agar [47, 49, 50], Gauze's medium with cycloheximide, nalidixic acid, novobiocin, and nystatin [51], M125 medium [49], tap water agar and ISP medium 2 [48], Reasoner's 2A agar (R2A) [2], GC agar [52], serum broth [52], and M1 agar plate [53].

\section{Isolation methods for Segniliparus spp.}

For the genus Segniliparus, the use of Middlebrook 7H10 and 7H11 media [3], Lowenstein-Jensen (LJ) medium [54], LJ with 5\% sodium chloride [54] and American Trudeau Society (ATS) media [54] have been suggested for isolation, good growth, and examination of morphological characteristics. Also, Segniliparus rugosus can grow on MacConkey agar [3] and it has been reported that $S$. rugosus is resistant to decontamination methods such as $\mathrm{NaOH}$ and $\mathrm{N}$-acetyl-L-cysteine in clinical specimens [55].

\section{Phenotypic identification of Williamsia}

Phenotypic characterizations are the first step for these bacteria identification at the genus and species levels. In the Williamsia there is 12 species names validly published includes Williamsia aurantiacus [53], W. deligens [40], Williamsia faeni [51], Williamsia herbipolensis [50], Williamsia limnetica [47], Williamsia maris [44], Williamsia marianensis [45], Williamsia muralis [2], Williamsia phyllosphaerae [49], Williamsia serinedens [43], Williamsia spongiae [46], and Williamsia sterculiae [48]. The species of this genus are distributed in different environments; however, they have also been isolated from clinical specimens [40]. Various phenotypic tests are properties of colonial morphology and pigment production (pigment colors in Williamsia spp. are yellow to orange or red [1]), producing aerial hyphae (this phenotypic characterization is seen in Williamsia, Skermania, Nocardia and, Millisia [4]), Gram stain (the genus Williamsia is Gram-positive), acid-fast stain (the genus Williamsia is not acid-fast [56]), hydrolysis of amino acids, acid production of carbohydrates, high-performance liquid chromatography (HPLC), gas-liquid chromatography (GLC), thin-layer chromatography (TLC) procedures, and enzymes production $[3,4,39,56]$. Some of the phenotypic characterization of the Williamsia spp. are shown in Table 1. Conventional phenotypic methods are unreliable and insufficient for differentiation of Williamsia and Segniliparus of related aerobic actinomycetes; therefore, molecular techniques have been used for accurate identification at the genus and species level. The temperature range for the growth for Williamsia spp. including $W$. limnetica, W. sterculiae, W. maris, W. muralis is 10 to $37^{\circ} \mathrm{C}[2,44,47,48]$, for $W$. aurantiacus and $W$. spongiae is 10 to $45^{\circ} \mathrm{C}[46,53]$, for $W$. phyllosphaerae and $W$. herbipolensis is 25 to $30{ }^{\circ} \mathrm{C}[49,50]$, for $W$. deligens is $37{ }^{\circ} \mathrm{C}$ [40], for $\mathrm{W}$. serinedens is 22 to $30{ }^{\circ} \mathrm{C}$ [43], for $\mathrm{W}$. marianensis is 4 to $30{ }^{\circ} \mathrm{C}$ [45], and for $W$. faeni is 10 to $30{ }^{\circ} \mathrm{C}$ [51]. Also, the $\mathrm{pH}$ range for the growth is between 4.0 and 10.0 for $W$. aurantiacus and $W$. spongiae $[46,53]$ and 5.0-8.0 for W. sterculiae [48].

\section{Phenotypic identification of Segniliparus}

Two species of Segniliparus rotundus and S. rugosus belong to the Segniliparus genus [3]. Its species are distributed in different environments; however, they have also been isolated from clinical specimens [57]. Pigment 


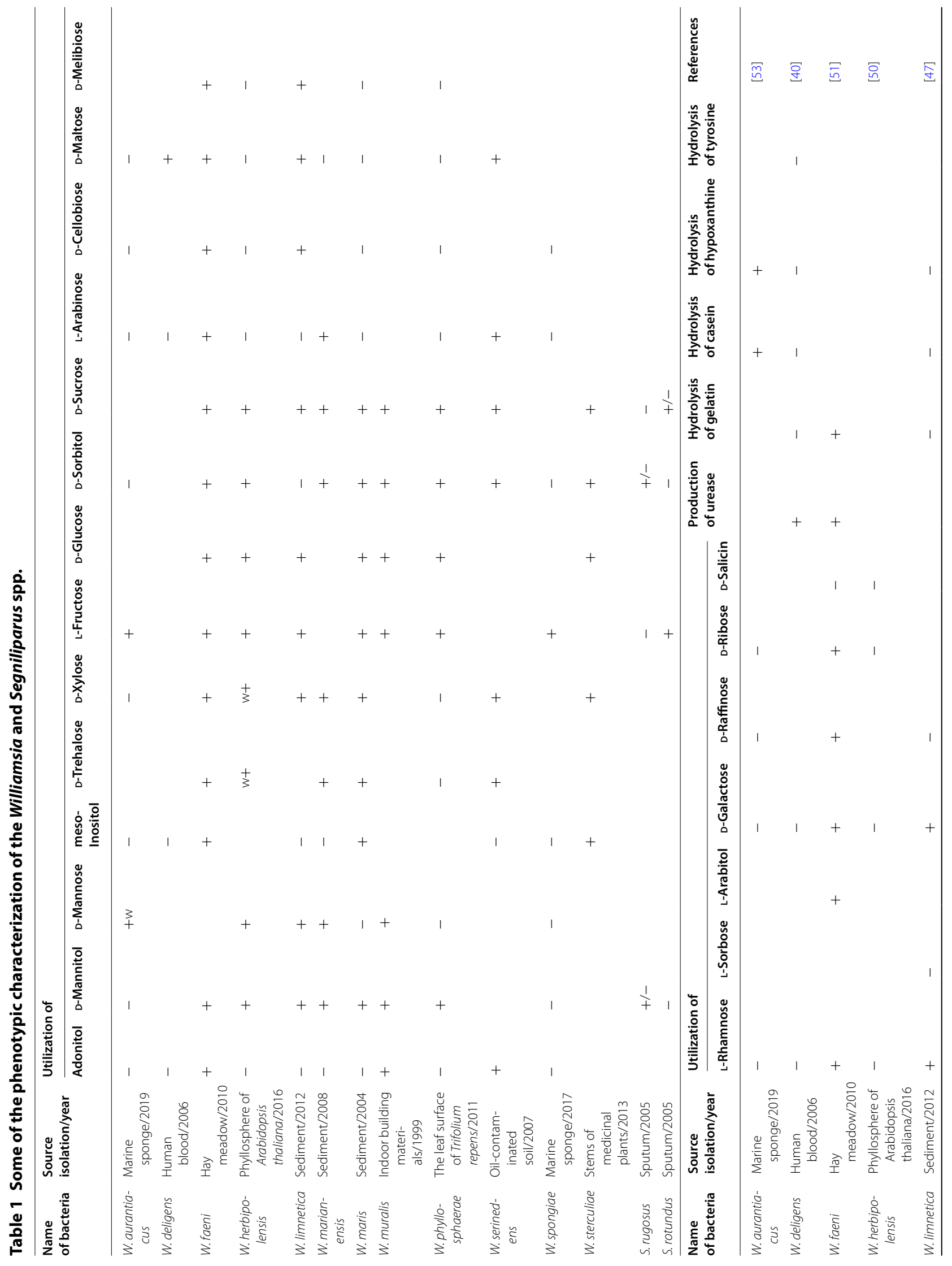




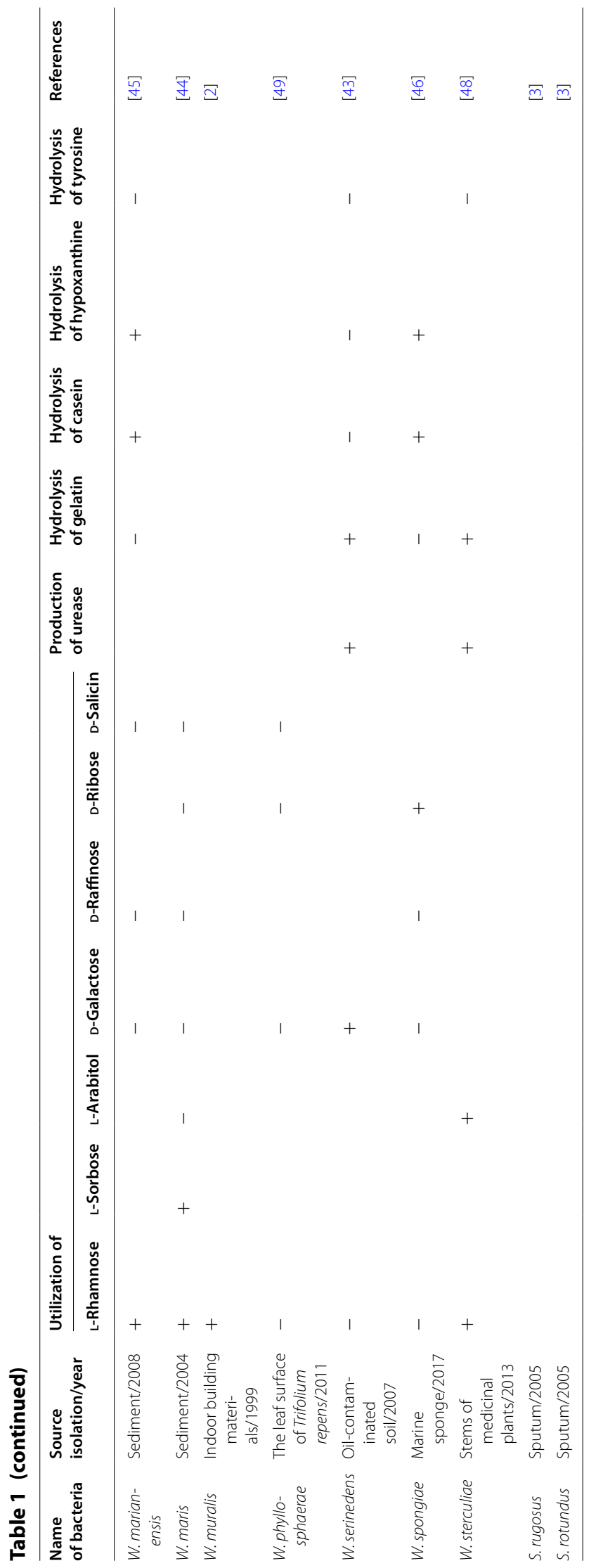


colors in Segniliparus spp. is white to beige [3]. An aerial hyphae are not seen in the Segniliparus [4]; and the genus is acid-fast [56]. Some of the phenotypic characterization of the Segniliparus spp. are shown in Table 1. The temperature range for the growth in Segniliparus spp. are as follows: S. rotundus: 28 to $37^{\circ} \mathrm{C}$ [3] and S. rugosus: 22 to $42{ }^{\circ} \mathrm{C}$ [3]. Researchers, medical laboratory personnel, and clinicians should note that in pulmonary specimens, especially in cystic fibrosis patients, the genus Segniliparus is similar to the genus Mycobacterium in acid-fast staining [54].

\section{Molecular identification of the Williamsia}

The most common molecular method for Williamsia accurate identification and assessment of taxonomic characteristics is sequence-based identification. 16S rRNA gene sequencing is an effective standard method for accurate identification of the novel bacteria and emerging pathogens at the genus and species levels [58]. Primers to amplify 16S rRNA gene for Williamsia identification include 27F (5'-AGAGTTTGATCCTGGCTC AG-3')/1492R (5'-GGTTACCTTGTTACGACTT-3') and $27 f\left(5^{\prime}\right.$-GAGTM'GATCCTGGCTCAG-3')/1525r (5' ${ }^{\prime}$-AGA AAGGAGGTGATCCAGCC-3') [40, 44]. Montoya-Porras et al. [59] identified the genus Williamsia with 454 pyrosequencing for the variable region of the 16S rRNA gene. The phylogenetic tree of the 16S rRNA gene for Williamsia standard species is shown in Fig. 1. The gold standard method to discern bacterial species is DNA-DNA hybridization (DDH) [30]; however, this method is not used in clinical laboratories for bacterial identification. Another molecular method is the whole-genome sequencing (WGS), which has been deposited for five Williamsia species in the National Center for Biotechnology Information (NCBI). Data are provided in Additional file 1.

\section{Molecular identification of the Segniliparus}

16S rRNA gene primers, such as $5^{\prime}$-GAGAGTTTG ATCCTG GCTCAG-3' $/ 5^{\prime}$ - AAG GAGGTG ATCCAG CCGCA-3' [3]; 8FPL (5'-AGTTTGATCCTGGCTCAG $\left.-3^{\prime}\right) / 806 \mathrm{R}$ (5'-GGACTACCAGGGTATCTAAT- $\left.3^{\prime}\right)$, and 515FPL (5'-TGCCAGCAGCCGCGGTAA-3')/13B (5'AGGCCCGGGAACGTATTCAC-3') have been used for Segniliparus identification [60]. The phylogenetic tree of the 16S rRNA gene for Segniliparus standard species is shown in Fig. 1. Butler et al. [3] reported that three of the four isolates of Segniliparus were not amplified for the $65 \mathrm{kDa}$ heat-shock protein ( $h s p 65$ gene) with TB11 (5'-ACCAACGATGGTGTGTCCAT-3') and TB12 (5'CTTGTCGAACCGCATACCCT-3') primers. Also, the cholesterol oxidase gene (choE gene) (this gene is a virulence factor gene in Rhodococcus equi) was not amplified for Segniliparus [3]. Koh et al. [55] used PCR-restriction fragment length polymorphism analysis (PRA) of the hsp65 (527-bp) [F: 5'-GAGGGCGTCATCACCGTC GAGG-3'/R: 5'-CGGCGATGGCGTCGGAGTCACC-3'] and $r p o B$ (360-bp) [F: 5'-TCAAGGAGAAGCGCTACG A-3'/R: 5'-GGATGTTGATCAGGGTCTGC-3'] genes for Segniliparus spp. identification. WGS of two Segniliparus isolates has been deposited in the National Center for Biotechnology Information (NCBI). Data are provided in Additional file 1.

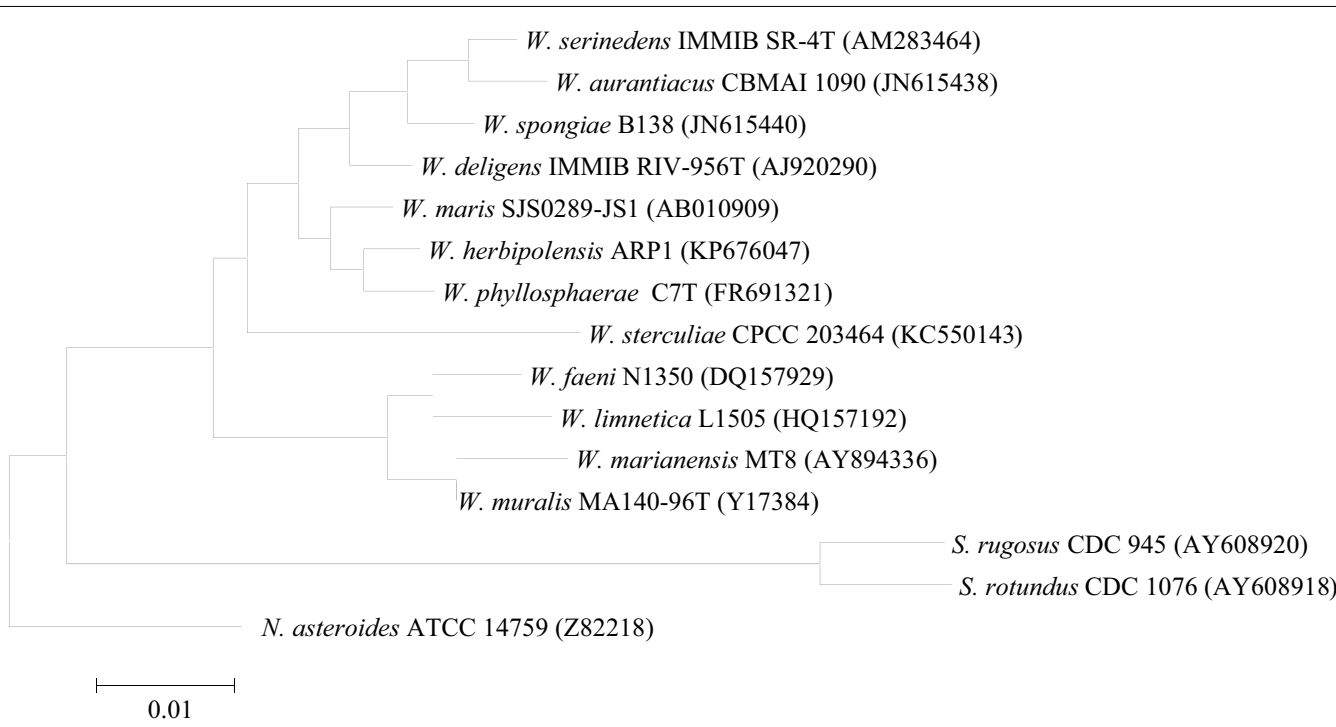

Fig. 1 The 16S rRNA gene-based phylogenetic tree of standard Williamsia and Segniliparus spp. with using the molecular evolutionary genetics analysis (MEGA) 5.0 software [66] which computed by the neighbor joining ( $\mathrm{NJ}$ ) analyses and kimura 2-parameter (K2P) model. The sequences were downloaded from NCBI. W: Williamsia, N: Nocardia, S: Segniliparus 
Table 2 Case reports published of Williamsia spp. in literature

\begin{tabular}{lllllll}
\hline Age/sex/country & Underlying disease & Type of infection & Isolated from & Name of organism & Outcome & References \\
\hline 66/M/Australia & Diabetic & Endophthalmitis & Vitreous fluid & W. muralis & Cure & [52] \\
80/F/Spain & $\begin{array}{l}\text { Allergy to penicillin and } \\
\text { high blood pressure }\end{array}$ & Lung infection & Brush & W. muralis & Died & [61] \\
31/F/Germany & Pregnant & Perinatal sepsis & Blood & W. serinedens & Cure & [62] \\
\hline
\end{tabular}

W. deligens isolated from blood [40] but case history is not available

Table 3 Case reports published of Segniliparus spp. in literature

\begin{tabular}{|c|c|c|c|c|c|c|}
\hline Age/sex/country & Underlying disease & Type of infection & Isolated from & Name of organism & Outcome & References \\
\hline /M/USA & Cystic fibrosis & Lung infection & Sputum & S. rugosus & Cure & {$[64]$} \\
\hline /M/USA & Cystic fibrosis & Lung infection & Sputum & S. rugosus & Cure & {$[64]$} \\
\hline 28/M/USA & Cystic fibrosis & Lung infection & $\mathrm{BAL}$ & S.rugosus & Cure & {$[64]$} \\
\hline Teenager/F/Australia & Cystic fibrosis & Lung infection & Sputum & S. rugosus & & {$[54]$} \\
\hline 43/F/South Korea & Immunocompetent & Lung infection & Sputum & S. rugosus & Cure & {$[55]$} \\
\hline 47/F/Korea & Immunocompetent & Lung infection & Sputum & S. rugosus & Cure & {$[60]$} \\
\hline
\end{tabular}

Segniliparus rotundus and Segniliparus rugosus isolated from sputum [3] but case history is not available

\section{Pathogenesis in Williamsia and Segniliparus}

Our knowledge about pathogenesis and virulence factors in two genera is limited. Cell wall components, such as mycolic acid, phagolysosome inhibition, immune response promotes, and the production of enzymes, such as catalase, may play a role in their pathogenesis.

\section{Clinical disease, antibiogram and treatment associated with Williamsia}

Physicians need to pay attention to these symptoms such as bilateral alveolar infiltrates [61], fever [62], having an underlying disease such as diabetes mellitus for detection of this rare infection [52]. Infections in humans caused by Williamsia have been reported. Infection occurs as a result of exposure to the environment; however, there is no evidence of an environmental source for Williamsia and Segniliparus infections. For antimicrobial susceptibility testing (AST), breakpoints have not been established for these genera, and researchers use recommended AST (the gold standard for antibiogram is micro broth dilution) for Nocardia and related aerobic actinomycetes by the Clinical and Laboratory Standards Institute (CLSI) [63]. Tomas et al. [61] first reported $W$. muralis as the cause of lung infection in an old woman. In their study, this bacterium was isolated from a brush sample and results of AST showed that this bacterium was susceptible to amoxicillin-clavulanate, cephalosporin (cefotaxime), carbapenem (imipenem), Quinolone (ciprofloxacin), aminoglycoside (tobramycin, gentamicin), sulfonamide (cotrimoxazole) and resistant to beta-lactam (ampicillin) and macrolide (erythromycin) family. In another study by Yassin et al. [40] reported $W$. deligens of human blood in 2006. Also, W. serinedens has been isolated of perinatal sepsis from a pregnant woman in 2010 and this bacterium was susceptible to amikacin, ampicillin, doxycycline, imipenem, linezolid, meropenem, penicillin $\mathrm{G}$, tobramycin, vancomycin and was resistant to oxacillin and trimethoprim-sulfamethoxazole with E-test method [62]. The case reports published regarding Williamsia spp. are provided in Table 2.

\section{Clinical disease, antibiogram and treatment associated with Segniliparus}

Physicians should pay more attention to symptoms such as chronic cough and sputum more than 3 months, fever, multiple small nodules in lung $[55,60]$ and radiologic finding similar to other genera in actinomycete family such as $M$. tuberculosis, non-tuberculous mycobacteria (NTM), and Nocardia for detection of this rare infection. Several studies have reported infections in humans caused by Segniliparus. The first report of the Segniliparus isolation from the clinical sample was published in 2005 by Butler et al. They isolated $S$. rugosus and $S$. rotundus from sputum and was AST performed using micro broth dilution. The results of AST showed that $S$. rotundus was susceptible to amikacin, cefoxitin, clarithromycin, ciprofloxacin, doxycycline, imipenem, sulfamethoxazole, and S. rugosus was susceptible to amikacin and sulfamethoxazole and resistant to clarithromycin, doxycycline, and tobramycin [3]. Butler et al. [64] isolated S. rugosus from 3 cystic fibrosis patients in 2007. Moreover, a study by Hansen et al. [54] isolated S. rugosus from sputum in a female with cystic fibrosis, and AST using micro 
broth dilution showed that this isolate was susceptible to ciprofloxacin, gatifloxacin, imipenem and resistant to amikacin, cefoxitin, ceftriaxone, tobramycin. Koh et al. [55] isolated S. rotundus from sputum in 2011 in a patient treated with clarithromycin and ciprofloxacin. In another study, $S$. rugosus was isolated from sputum in 2014 [60]. S. rugosus possibly is an emerging pathogen in cystic fibrosis patients. Antibiotic resistance genes have not been reported in the genera Williamsia and Segniliparus [65]. The case reports published on Segniliparus spp. are provided in Table 3. On the basis of the clinical reports, the pulmonary infection of Segniliparus spp. is associated with chronic cough, fever and hypoventilation, as well as the presence of multiple small nodules, with symptoms of acid-fast bacilli in sputum and radiologic results similar to $M$. tuberculosis, NTM, Nocardia and so on. Therefore, pulmonary infection should be identified in microbiology laboratories.

\section{Conclusion}

In this review, we surveyed taxonomy and the role of the genera Williamsia and Segniliparus in human infections. The identification of pathogenic factors in these bacteria requires more investigations. A few studies have been conducted on Williams and Segniliparus infections because of the lack of attention and insufficient experience in medical laboratory personnel as well as the lack of optimization of the phenotypic and molecular methods to identify these bacteria in hospitals. The use of novel molecular methods is necessary for accurate identification of Williamsia and Segniliparus species.

\section{Supplementary Information}

The online version contains supplementary material available at https://doi. org/10.1186/s12941-021-00416-z.

Additional file 1. Whole genome sequence data of Williamsia and Segniliparus spp.

\section{Acknowledgements}

The authors would like to thank of Shahid Sadoughi University of Medical Sciences for their support.

\section{Authors' contributions}

MFB participated in the design and drafting of the manuscript. The author read and approved the final manuscript.

\section{Ethics approval and consent to participate}

This research does not contain any human or animal participants performed by any of the authors.

\section{Competing interests}

The authors declare that they have no competing interests.

Received: 26 August 2020 Accepted: 12 January 2021

Published online: 23 January 2021
References

1. Mochon AB, Sussland D, Saubolle MA. Aerobic actinomycetes of clinical significance. Microbiol Spectr. 2016. https://doi.org/10.1128/microbiols pec.DMIH2-0021-2015.

2. Kämpfer P, Andersson MA, Rainey FA, Kroppenstedt RM, SalkinojaSalonen M. Williamsia muralis gen. nov., sp. nov., isolated from the indoor environment of a children's day care centre. Int J Syst Evolut Microbiol. 1999;49(2):681-7.

3. Butler WR, Floyd MM, Brown JM, Toney SR, Daneshvar MI, Cooksey RC, et al. Novel mycolic acid-containing bacteria in the family Segniliparaceae fam. Nov., including the genus Segniliparus gen. nov., with descriptions of Segniliparus rotundus sp. nov. and Segniliparus rugosus sp. nov. Int J Syst Evolut Microbiol. 2005;55(4):1615-24.

4. Kämpfer P. Williamsia. In: Bergey's manual of systematics of Archaea and Bacteria. New York: Springer; 2015. p. 1-7.

5. Tindall B. On the status of the names Corynebacteriales Goodfellow and Jones 2015, Mycobacteriales Janke 1924 (Approved Lists 1980) and Mycobacteriales Cavalier-Smith 2002. Int J Syst Evolut Microbiol. 2019;69(10):3310-2.

6. Kalt F, Schulthess B, Sidler F, Herren S, Fucentese SF, Zingg PO, et al. Corynebacterium species rarely cause orthopedic infections. J Clin Microbiol. 2018;56:e01200-18.

7. Bernard K. The genus Corynebacterium and other medically relevant coryneform-like bacteria. J Clin Microbiol. 2012;50(10):3152-8.

8. Tauch A, Fernández-Natal I, Soriano F. A microbiological and clinical review on Corynebacterium kroppenstedtii. Int J Infect Dis. 2016;48:33-9.

9. Andalibi F, Fatahi-Bafghi M. Gordonia: isolation and identification in clinical samples and role in biotechnology. Folia Microbiol. 2017;62(3):245-52.

10. Gueneau R, Blanchet D, Rodriguez-Nava V, Bergeron E, Soulier M, Bestandji N, et al. Actinomycetoma caused by Gordonia westfalica: first reported case of human infection. New Microbes New Infect. 2020;34:100658.

11. Porvaznik I, Solovič I, Mokrý J. Non-tuberculous mycobacteria: classification, diagnostics, and therapy. In: Respiratory treatment and prevention. Cham: Springer; 2016. p. 19-25.

12. Bouam A, Heidarieh P, Shahraki AH, Pourahmad F, Mirsaeidi M, Hashemzadeh $\mathrm{M}$, et al. Mycobacterium ahvazicum sp. nov., the nineteenth species of the Mycobacterium simiae complex. Sci Rep. 2018;8(1):1-12.

13. Forbes BA. Mycobacterial taxonomy. J Clin Microbiol. 2016:55:380-3.

14. Tortoli E. Microbiological features and clinical relevance of new species of the genus Mycobacterium. Clin Microbiol Rev. 2014;27(4):727-52.

15. Navid S, Sadegh-Ehdaei B, Shabani M, Hasani M, Mirzaei A, Ghazvini K, et al. The case report of Mycobacterium arupense wound infection in diabetes mellitus patients; the first report and literature review. Access Microbiol. 2020;2(4):e000106.

16. Supply P, Brosch R. The biology and epidemiology of Mycobacterium canettii. In: Strain variation in the Mycobacterium tuberculosis complex: its role in biology, epidemiology and control. Cham: Springer; 2017. p. 27-41.

17. Riccardi N, Monticelli J, Antonello RM, Luzzati R, Gabrielli M, Ferrarese M, et al. Mycobacterium chimaera infections: an update. J Infect Chemother. 2020;26(3):199-205.

18. Dastranj M, Farahani A, Hashemi Shahraki A, Atashi S, Mohajeri P. Molecular identification and distribution of non-tuberculous mycobacteria isolated from clinical specimens by PCR-sequencing method in West of Iran. Clin Respir J. 2018;12(3):996-1002.

19. Gharbi R, Mhenni B, Fraj SB, Mardassi H. Nontuberculous mycobacteria isolated from specimens of pulmonary tuberculosis suspects, Northern Tunisia: 2002-2016. BMC Infect Dis. 2019;19(1):819.

20. litoh E, Tominaga M, Okamoto M, Sakazaki Y, Nakamura M, Kinoshita T, et al. A case of pulmonary Mycobacterium heckeshornense infection in a healthy Japanese man. Respir Med Case Rep. 2020. https://doi.org/10.1016/j. rmcr.2020.101093.

21. Pouresmaeil O, Fatahi-Bafghi M, Safaei S. The role of nontuberculous mycobacteria in cutaneous infections of diabetic patients: a literature review. Iran J Dermatol. 2018;21(1):14-9.

22. Gopalaswamy R, Shanmugam S, Mondal R, Subbian S. Of tuberculosis and non-tuberculous mycobacterial infections - a comparative analysis of epidemiology, diagnosis and treatment. J Biomed Sci. 2020;27(1):1-17.

23. Mv RAO. Infection of Mycobacterium mageritense at surgical site: a first case report of India. J Drug Deliv Ther. 2019;9(1-s):369-73. 
24. Johnson MG, Stout JE. Twenty-eight cases of Mycobacterium marinum infection: retrospective case series and literature review. Infection. 2015;43(6):655-62

25. Coolen-Allou N, Touron T, Belmonte O, Gazaille V, Andre M, Allyn J, et al. Clinical, radiological, and microbiological characteristics of Mycobacterium simiae infection in 97 patients. Antimicrob Agents Chemother. 2018;62(7):e0039518.

26. Wei M, Zhao Y, Qian Z, Yang B, Xi J, Wei J, et al. Pneumonia caused by Mycobacterium tuberculosis. Microbes Infect. 2020:22(6-7):278-84.

27. Steffen CM, Freeborn H. Mycobacterium ulcerans in the Daintree 2009-2015 and the mini-epidemic of 2011. ANZ J Surg. 2018;88(4):E289-93.

28. Teng JL, Fong JY, Fok KM, Lee HH, Chiu TH, Tang Y, et al. Tsukamurella asaccharolytica sp. nov., Tsukamurella conjunctivitidis sp. nov. and Tsukamurella sputi sp. nov., isolated from patients with bacteraemia, conjunctivitis and respiratory infection in Hong Kong. Int J Syst Evolut Microbiol. 2020;70(2):995-1006.

29. Safaei S, Fatahi-Bafghi M, Pouresmaeil O. Role of Tsukamurella species in human infections: first literature review. New Microbes New Infect. 2018;22:6-12.

30. Fatahi-Bafghi M. Nocardiosis from 1888 to 2017. Microb Pathog. 2018;114:369-84.

31. Gnanam H, Rajapandian SGK, Gunasekaran R, Prithiviraj SR, Ravindran RS, Sen S, et al. Molecular identification of Nocardia species causing endophthalmitis using multilocus sequence analysis (MLSA): a 10-year perspective. J Med Microbiol. 2020;69(5):728-38.

32. Nouioui I, Cortés-Albayay C, Neumann-Schaal M, Vicente D, Cilla G, Klenk $\mathrm{H}-\mathrm{P}$, et al. Genomic virulence features of two novel species Nocardia barduliensis sp. nov. and Nocardia gipuzkoensis sp. nov., isolated from patients with chronic pulmonary diseases. Microorganisms. 2020;8(10):1517.

33. Ito $Y$, Tanigawa M, Yaguchi T, Toyoshima H, Iwamoto K, Nigi A, et al. Pulmonary nocardiosis caused by Nocardia blacklokiae in an immunocompetent patient. Respir Med Case Rep. 2020;29:101005

34. Hashemi-Shahraki A, Heidarieh P, Bostanabad SZ, Hashemzadeh M, Feizabadi MM, Schraufnagel D, et al. Genetic diversity and antimicrobial susceptibility of Nocardia species among patients with nocardiosis. Scic Rep. 2015;5:17862

35. Zhou T, Wang X-Y, Deng D-Q, Xu L-H, Li X-L, Guo Y, et al. Nocardia colli sp. nov., a new pathogen isolated from a patient with primary cutaneous nocardiosis. Int J Syst Evolut Microbiol. 2020;70(5):2981-7.

36. Rahdar HA, Gharabaghi MA, Bahador A, Shahraki-Zahedani S, KaramiZarandi M, Mahmoudi S, et al. Pulmonary Nocardia ignorata infection in Gardener, Iran, 2017. Emerg Infect Dis. 2020;26(3):610.

37. Ercibengoa M, Càmara J, Tubau F, García-Somoza D, Galar A, Martín-Rabadán P, et al. A multicentre analysis of Nocardia pneumonia in Spain: 2010-2016. Int J Infect Dis. 2020;90:161-6.

38. Majidzadeh M, Fatahi-Bafghi M. Current taxonomy of Rhodococcus species and their role in infections. Eur J Clin Microbiol Infect Dis. 2018;37(11):2045-62.

39. Whitman WB, Goodfellow M, Kämpfer P. Bergey's manual of systematic bacteriology: Volume 5: the Actinobacteria. New York: Springer; 2012.

40. Yassin A, Hupfer H. Williamsia deligens sp. nov., isolated from human blood. Int J Syst Evolut Microbiol. 2006;56(1):193-7.

41. Marrakchi H, Lanéelle M-A, Daffé M. Mycolic acids: structures, biosynthesis, and beyond. Chem Biol. 2014;21(1):67-85.

42. Falkinham JO. Common features of opportunistic premise plumbing pathogens. Int J Environ Res Public Health. 2015;12(5):4533-45.

43. Yassin A, Young CC, Lai W-A, Hupfer H, Arun A, Shen F-T, et al. Williamsia serinedens sp. nov., isolated from an oil-contaminated soil. Int J Syst Evolut Microbiol. 2007;57(3):558-61.

44. Stach JE, Maldonado LA, Ward AC, Bull AT, Goodfellow M. Williamsia maris sp. nov., a novel actinomycete isolated from the Sea of Japan. Int J Syst Evolut Microbiol. 2004;54(1):191-4.

45. Pathom-Aree W, Nogi Y, Sutcliffe IC, Ward AC, Horikoshi K, Bull AT, et al. Williamsia marianensis sp. nov., a novel actinomycete isolated from the Mariana Trench. Int J Syst Evolut Microbiol. 2006;56(5):1123-6.
46. de Menezes CBA, Afonso RS, de Souza WR, Parma M, de Melo IS, Zucchi TD, et al. Williamsia spongiae sp. nov., an actinomycete isolated from the marine sponge Amphimedon viridis. Int J Syst Evolut Microbiol. 2017;67(5):1260-5.

47. Sazak A, Sahin N. Williamsia limnetica sp. nov., isolated from a limnetic lake sediment. Int J Syst Evolut Microbiol. 2012;62(6):1414-8.

48. Fang X-M, Su J, Wang H, Wei Y-Z, Zhang T, Zhao L-L, et al. Williamsia sterculiae sp. nov., isolated from a Chinese medicinal plant. Int J Syst Evolut Microbiol. 2013;63(11):4158-62.

49. Kämpfer P, Wellner S, Lohse K, Lodders N, Martin K. Williamsia phyllosphaerae sp. nov., isolated from the surface of Trifolium repens leaves. Int J Syst Evolut Microbiol. 2011;61(11):2702-5.

50. Kämpfer P, Busse H-J, Horn H, Abdelmohsen UR, Hentschel U, Glaeser SP. Williamsia herbipolensis sp. nov., isolated from the phyllosphere of Arabidopsis thaliana. Int J Syst Evolut Microbiol. 2016;66(11):4609-13.

51. Jones A, Payne G, Goodfellow M. Williamsia faeni sp. nov., an actinomycete isolated from a hay meadow. Int J Syst Evolut Microbiol. 2010;60(11):2548-51.

52. Murray RJ, Aravena-Roman M, Kämpfer P. Endophthalmitis due to Williamsia muralis. J Med Microbiol. 2007;56(10):1410-2.

53. de Menezes CBA, Afonso RS, de Souza WR, Parma MM, de Melo IS, Fugita FLS, et al. Williamsia aurantiacus sp. nov. a novel actinobacterium producer of antimicrobial compounds isolated from the marine sponge. Arch Microbiol. 2019;201(5):691-8.

54. Hansen T, Van Kerckhof J, Jelfs P, Wainwright C, Ryan P, Coulter C. Segniliparus rugosus infection, Australia. Emerg Infect Dis. 2009;15(4):611.

55. Koh W-J, Choi G-E, Lee S-H, Park YK, Lee NY, Shin SJ. First case of Segniliparus rotundus pneumonia in a patient with bronchiectasis. J Clin Microbiol. 2011:49(9):3403-5.

56. Procop GW, Church D, Hall G, Janda W. Koneman's color atlas and textbook of diagnostic. Philadelphia: Wolters Kluwer; 2017.

57. Earl AM, Desjardins CA, Fitzgerald MG, Arachchi HM, Zeng Q, Mehta T, et al. High quality draft genome sequence of Segniliparus rugosus CDC 945 $\mathrm{T}=($ ATCC BAA-974 T). Stand Genom Sci. 2011:5(3):389.

58. Schlaberg R, Simmon KE, Fisher MA. A systematic approach for discovering novel, clinically relevant bacteria. Emerg Infect Dis. 2012;18(3):422.

59. Montoya-Porras LM, Omar T-C, Alzate JF, Moreno-Herrera CX, CadavidRestrepo GE. 16S rRNA gene amplicon sequencing reveals dominance of Actinobacteria in Rhodnius pallescens compared to Triatoma maculata midgut microbiota in natural populations of vector insects from Colombia. Acta Trop. 2018;178:327-32.

60. Lee JY, Chon GR, Sung H, Shim TS, Jo K-W. A case of Segniliparus rugosus pulmonary infection in an immunocompetent patient with non-cystic fibrosis. Tuberc Respir Dis. 2014;77(5):227-9.

61. del MarTM, Moure R, Nieto JAS, Fojon S, Fernandez A, Diaz M, et al. Williamsia muralis pulmonary infection. Emerg Infect Dis. 2005;11(8):1324.

62. Yassin AF, Lombardi SJ, Fortunato SJ, McNabb PC, Carr MB, Trabue CH. Perinatal sepsis caused by Williamsia serinedens infection in a 31-year-old pregnant woman. J Clin Microbiol. 2010;48(7):2626-9.

63. Woods GL, Brown-Elliott BA, Conville PS, Desmond EP, Hall GS, Lin G, et al. Susceptibility testing of mycobacteria, Nocardiae, and other aerobic actinomycetes. Wayne: Clinical and Laboratory Standards Institute; 2011.

64. Butler WR, Sheils CA, Brown-Elliott BA, Charles N, Colin AA, Gant MJ, et al. First isolations of Segniliparus rugosus from patients with cystic fibrosis. J Clin Microbiol. 2007;45(10):3449-52.

65. Fatahi-Bafghi M. Antibiotic resistance genes in the Actinobacteria phylum. Eur J Clin Microbiol Infect Dis. 2019;38:1599-624.

66. Tamura K, Peterson D, Peterson N, Stecher G, Nei M, Kumar S. MEGA5: molecular evolutionary genetics analysis using maximum likelihood, evolutionary distance, and maximum parsimony methods. Mol Biol Evol. 2011:28(10):2731-9.

\section{Publisher's Note}

Springer Nature remains neutral with regard to jurisdictional claims in published maps and institutional affiliations. 